\title{
A MULTI-OBJECTIVE SCHEDULING OPTIMIZATION MODEL CONSIDERING PRODUCT BLOCKAGE AND MACHINE FAULTS
}

\author{
Yin, H. Y. ; Liu, L. Z. ${ }^{* * \#} \&$ Yeh, J. S. ${ }^{* * *}$ \\ *School of Computer and Information Engineering, Xiamen University of Technology, \\ Xiamen 361024, China \\ ** Post Doctoral Work Station of Jinjiang Science and Technology Park, Jinjiang 362200, China \\ ${ }^{* * * *}$ Department of Computer Science and Information Management, Providence University, \\ Taichung 43301, Taiwan \\ E-Mail: Huayi@xmut.edu.cn, 1lz@xmut.edu.cn (" Corresponding author), jsyeh@pu.edu.tw
}

\begin{abstract}
Considering problems like product blockage and machine faults in the batch scheduling of product assembly lines, this paper proposes a multi-objective flow shop batch scheduling model taking these problems into account, and by comparing with the traditional flow shop scheduling model, it verifies the feasibility and superiority of this model. The results show that, regarding the product blockage problem, this paper improves the MWH heuristic algorithm, leading to better quality of the initial population produced; and that it also proposes a new ISBOX crossover operator algorithm, which is used in combination with the nondominated solution information to calculate the new solution for offsprings, improving the local search capability of the algorithm. In order to address machine faults, this paper obtains new solutions by optimizing the maximum completion time and the early completion time, takes the new solutions as the initial population, and uses the proposed model to optimize the robustness and stability of the existing algorithm. By comparing the proposed model and the traditional scheduling model in terms of product blockage and machine fault, this paper finds that the proposed algorithm has high local and global convergence, and generates high-quality nondominated solutions. According to the re-scheduling strategy, this algorithm can effectively reduce the effects of product blockage and machine faults on the objective function.

(Received, processed and accepted by the Chinese Representative Office.)
\end{abstract}

Key Words: Flow Shop, Optimized Scheduling, Multi-Objective, Machine Fault, Blockage

\section{INTRODUCTION}

The traditional workshop assembly line scheduling is about allocating a certain amount of resources on demand to different tasks in the workshop to optimize the completion time and production efficiency of the entire operating system. The workshop assembly line scheduling mainly includes single-machine scheduling, job scheduling, parallel scheduling and so on.

In actual production, there are many uncertainties, such as product processing blockage, machine fault, uncertain production processes and so on. The production scheduling of assembly lines involves the combination and optimization of multiple factors. Currently, researchers have proposed a number of flow shop scheduling optimization schemes. By algorithm, these can be divided into classical algorithms, approximation algorithms and intelligent algorithms [1-10]. Classical algorithms include the dynamic programming method and branch and bound algorithm; the approximation algorithms include the simulation algorithm, NEH algorithm and Lagrangian relaxation algorithm; and intelligent algorithms include the annealing algorithm, tabu search algorithm and genetic algorithm [11-18]. These algorithms are very effective in solving specific problems, but all subject to some limitations.

So far, few studies have been carried out on the product blockage and machine faults occurring during the product processing on assembly lines [19-20]. When there is any machine fault, the original scheduling scheme may no longer be the optimal one and needs to 
be redesigned, which means, re-scheduling strategy needs to be used to address the uncertainties in flow shop scheduling brought by product blockage and machine faults [21-26].

Considering problems like product blockage and machine faults in the batch scheduling of product assembly lines, this paper proposes a multi-objective flow shop batch scheduling model taking these problems into account, and by comparing with the traditional flow shop scheduling model, it verifies the feasibility and superiority of this model.

\section{MULTI-OBJECTIVE FLOW SHOP SCHEDULING CONSIDERING BLOCKAGE EFFECT}

\subsection{Description of the scheduling model}

The multi-objective pipeline scheduling model considering the blocking effect can be expressed as follows.

Let the product sequence be:

$$
\pi=\{\pi(1), \pi(2), \pi(3), \ldots \pi(n)\}
$$

$\pi(j)$ can be further divided into several sub-products $\pi(j)=\left\{\pi(j)^{1}, \pi(j)^{2}, \pi(j)^{3}, \ldots, \pi(j)^{l}\right\}$. Product processing needs to meet the following conditions:

(a) Until all the sub-products of one product are finished, the next product will not be produced;

(b) A machine can only process one sub-product at the same time. There is no buffer area between adjacent machines;

(c) There is certain wait time between the processing of two products;

(d) The processing time includes product processing time, machine preparation time and sub-product transportation time.

As there is no product buffer area on any traditional product processing line, there will be products stopping on and jamming the line during the process. Therefore, the total processing time of a product should take into account the blocking time.

Set the objective functions as the minimum product completion time $f_{1}$ and maximum earliness $f_{2}$.

$$
\begin{gathered}
\min f_{1}=C_{\pi(n), m, l_{\pi(n)}} \\
f_{2}=\sum_{j=1}^{n} \max \left(0, d_{j}-C_{\pi(j), m, l_{\pi(j)}}\right)
\end{gathered}
$$

$l_{\pi(n)}$ is the number of sub-products within the product $n ; C_{\pi(n)}$ is the completion time of the product by the machine.

\subsection{Improved Hybrid Artificial Bee Colony Algorithm}

Hybrid Artificial Bee Colony Algorithm (HABC) has been proved to be effective when addressing multi-objective flow shop scheduling, but when the blockage effect is considered, the initialization strategy and the elite retention mechanism in HABC cannot meet the requirements. Therefore, based on the HABC algorithm, this paper proposes an improved hybrid artificial bee colony algorithm (IHABC) and uses the heuristic algorithm to generate multiple elite initial solutions, and then uses the nondominated solution design to obtain crossover operators and the multi-objective local search method.

This paper uses the vNEH heuristic algorithm to initialize the population and generate a number of high-quality initial solutions and uses the random function to randomly generate a solution in the search space. If the solution falls within the population set, the algorithm removes this solution and continues to the next calculation; otherwise, it will put the solution 
into the population. When the number of new solutions in the population is greater than the original number in the population, the algorithm terminates.

The traditional algorithm is random and aimless when generating new individuals, which increases the difficulty in the global convergence of the algorithm. The Improved Similar Job Order Crossover (ISJOX) is used to increase the proportion of elite individuals in the population: first, it generates a temporary solution to contain all nondominated solutions; secondly, it selects parent individual that has the same solution as that in the temporary solution set from the population for genetic operations; finally, it implements the repair strategy and uses the single-point cross method to obtain the remaining parts of the offspring. As the ISJOX algorithm is displacive, it is not limited by spatial position and can effectively prevent high-quality products from being damaged in the process.

\subsection{Steps of multi-objective flow shop scheduling}

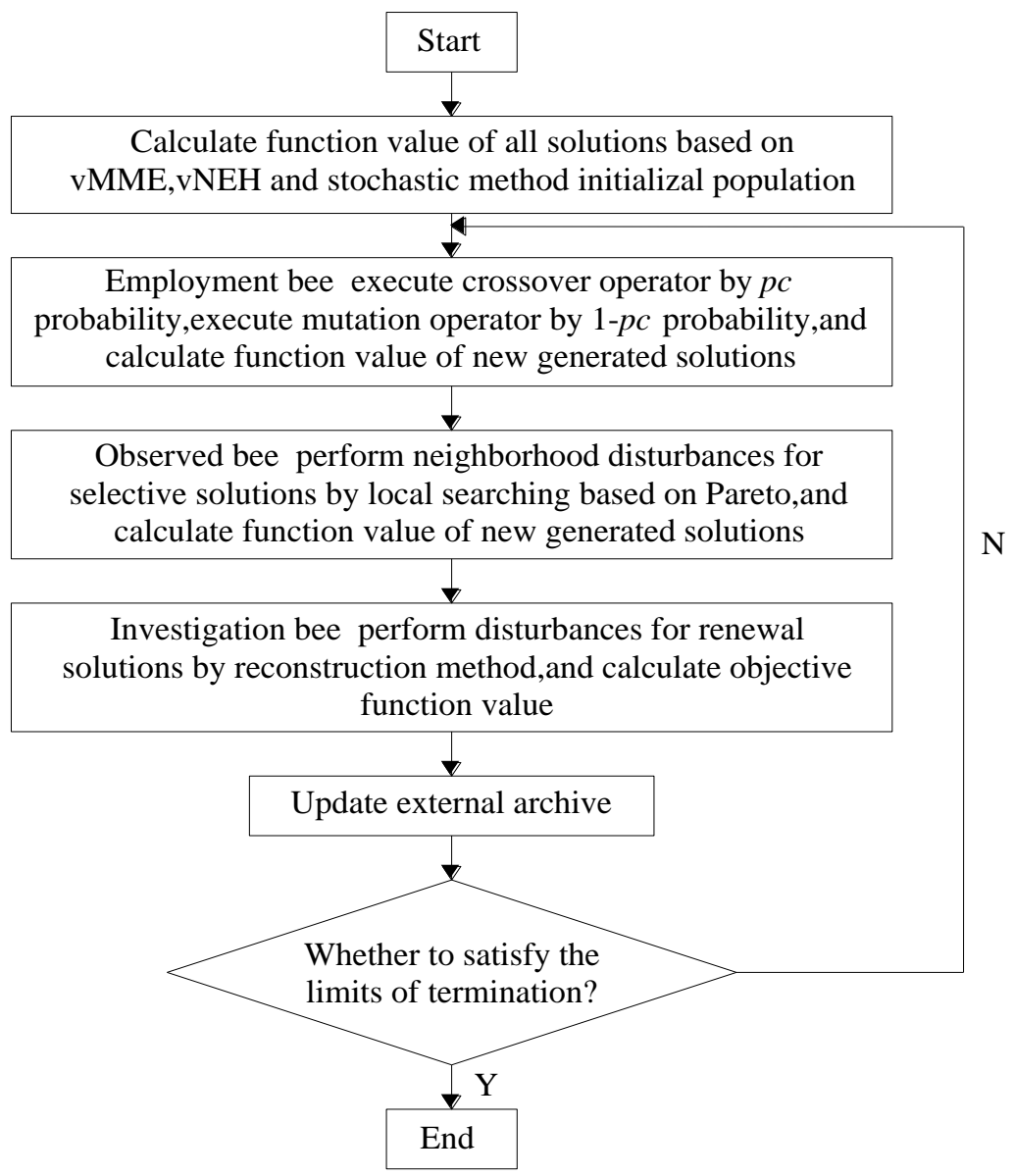

Figure 1: Flowchart of the multi-objective blocking lot-streaming flow shop scheduling.

According to the above theory, this paper establishes a flow shop scheduling model considering product blockage. The core steps of the algorithm are shown in Fig. 1 and outlined as follows:

(1) Set the population size, crossover probability, local search probability and iterative completion time;

(2) Use vNEH to initialize the population and calculate the objective functions for the new solutions;

(3) Use the local search function to search the neighbourhood of high-quality solutions in the population to enhance the development ability of the algorithm; 
(4) For solutions not updated, use the reconstruction algorithm to carry out forced disturbance updates;

(5) Use the EAs to store the nondominated solution obtained from each round of iteration. There will be many times of iterations (100-200 times). After 200 times of iterations, determine whether the condition for iteration termination is satisfied.

\subsection{Test and verification}

In order to verify the rationality of the proposed algorithm, this paper compares it with the traditional scheduling algorithm. The verification criteria are as follows.

Number of nondominated solutions $N\left(S_{i}\right)$ :

$$
N\left(S_{i}\right)=\left|x^{\prime} \in S_{i}\right| \exists x^{\prime} \prec \forall y, y \in S \bigcap x \neq y \mid \quad S=\underset{i=1,2,3,4}{\cup} S_{i}
$$

The greater $N\left(S_{i}\right)$ is, the more nondominated solutions the algorithm will have.

The proportion of nondominated solutions $R\left(S_{i}\right)$ is:

$$
R\left(S_{i}\right)=\frac{N\left(S_{i}\right)}{\left|S_{i}\right|}
$$

$R\left(S_{i}\right)$ can be used to evaluate the quality of the nondominated solution set. The greater the $R\left(S_{i}\right)$, the better the quality of the nondominated solutions.

Distance from the reference solution set $D\left(S_{i}\right)$ :

$$
\begin{gathered}
D\left(S_{i}\right)=\frac{1}{\left|S^{*}\right|} \sum_{y \in S^{*}} d_{y}\left(S_{i}\right) \\
d_{y}\left(S_{i}\right)=\min _{x \in S_{i}}\left\{\sqrt{\sum_{k=1}^{4}\left(\frac{f_{k}(x)-f_{k}(y)}{f_{k}^{\max }(\bullet)-f_{k}^{\min }(\bullet)}\right)^{2}}\right\}
\end{gathered}
$$

$D\left(S_{i}\right)$ is mainly used to evaluate the convergence of the algorithm. $S^{*}$ is the reference set; $f_{k}(x)$ and $f_{k}(y)$ are the $k^{\text {th }}$ objective functions of the independent variables. The larger the $D\left(S_{i}\right)$ is, the farther away the solution will be from the centre of the optimal solution.

Table I shows how the changes in the crossover operator pc affect the IHABC algorithm proposed in this paper. The $p c$ value is increased from 0 gradually to 0.8 .8 instances are used for comparison.

Table I: Sensitivity study of $p c$ with respect to the distance indicator.

\begin{tabular}{|c|ccccccccc|}
\hline Instance & \multicolumn{7}{|c|}{$p c$} \\
\hline$n \times m$ & 0 & 0.1 & 0.2 & 0.3 & 0.4 & 0.5 & 0.6 & 0.7 & 0.8 \\
\hline $50 \times 10$ & 0.29 & 0.22 & 0.07 & 0.03 & 0.04 & 0.16 & 0.06 & 0.28 & 0.14 \\
$50 \times 20$ & 0.31 & 0.17 & 0.16 & 0.06 & 0.04 & 0.21 & 0.07 & 0.09 & 0.02 \\
$70 \times 10$ & 0.27 & 0.19 & 0.24 & 0.10 & 0.15 & 0.44 & 0.05 & 0.12 & 0.11 \\
$70 \times 20$ & 0.26 & 0.21 & 0.11 & 0.12 & 0.19 & 0.28 & 0.12 & 0.05 & 0.08 \\
$90 \times 10$ & 0.33 & 0.15 & 0.26 & 0.15 & 0.07 & 0.06 & 0.02 & 0.06 & 0.05 \\
$90 \times 20$ & 0.25 & 0.23 & 0.15 & 0.24 & 0.16 & 0.24 & 0.07 & 0.22 & 0.27 \\
$110 \times 10$ & 0.47 & 0.41 & 0.31 & 0.52 & 0.18 & 0.21 & 0.09 & 0.20 & 0.25 \\
$110 \times 20$ & 0.28 & 0.38 & 0.33 & 0.22 & 0.18 & 0.19 & 0.13 & 0.20 & 0.42 \\
mean & 0.32 & 0.29 & 0.18 & 0.16 & 0.15 & 0.19 & 0.08 & 0.16 & 0.19 \\
\hline
\end{tabular}

From the table, it can be seen that, when $p c=0$, the proposed algorithm only generates new solutions through the mutation operator and it does not involve any crossover operation; on the whole, the distance value $D\left(S_{i}\right)$ first decreases and then increases along with the increase of $p c$; when $p c=0.6$, the distance value $D\left(S_{i}\right)$ reaches the minimum, indicating that 
the traditional solution mainly performs the crossover operator and has a very small probability of performing the mutation operator, leading to local optimal solutions. The algorithm proposed in this paper increases the probability of the new solution performing the mutation operator and improves the perturbation of the solution, leading to a higher probability of a global optimal solution, so the $p c$ value is set to 0.6.

Table II shows the comparison results of $N\left(S_{i}\right), D\left(S_{i}\right)$ and $R\left(S_{i}\right)$ under the three initialization strategies. The three initialization strategies are random generation of initial population (Random), NEH algorithm and the proposed $\mathrm{vNEH}$ initialization algorithm (the proposed method). From the table, it can be seen that in terms of $N\left(S_{i}\right)$, there are 2 nondominant solutions obtained by the initialization function under the random algorithm, 3 under the NEH algorithm and 6 under the proposed method; in terms of $D\left(S_{i}\right)$, the value calculated by the proposed method is far less than those by the other two algorithms; in terms of $R\left(S_{i}\right)$, the average $R\left(S_{i}\right)$ value by the proposed algorithm is $77 \%$, and the $R\left(S_{i}\right)$ values by the other two algorithms are more than $50 \%$, indicating that the initialization algorithm proposed in this paper is more superior - the initial population is of high quality and the convergence is fast.

Table II: Performance of different initialization strategies.

\begin{tabular}{|c|ccc|cccc|ccc|}
\hline Instance & \multicolumn{4}{|c|}{$N(S)$} & \multicolumn{3}{c|}{$R(S)(\%)$} & \multicolumn{3}{c|}{$D(S)$} \\
\hline$n \times m$ & Random & NEH+M & propos. & Random & NEH+M & propos. & Random & NEH+M & propos. \\
\hline $50 \times 10$ & 0.39 & 0.29 & 4.44 & 100.0 & 54.00 & 55.00 & 3.63 & 1.12 & 0.14 \\
$50 \times 20$ & 3.22 & 1.02 & 0.35 & 0.00 & 3.67 & 1.14 & 0.54 & 0.46 & 0.00 \\
$70 \times 10$ & 0.27 & 5.31 & 8.27 & 50.00 & 44.00 & 100.0 & 0.46 & 0.25 & 0.10 \\
$70 \times 20$ & 2.24 & 2.37 & 7.47 & 65.00 & 80.00 & 100.0 & 0.22 & 0.05 & 0.00 \\
$90 \times 10$ & 2.51 & 1.28 & 4.24 & 100.0 & 90.00 & 55.00 & 0.00 & 0.16 & 0.01 \\
$90 \times 20$ & 2.44 & 3.45 & 7.29 & 0.00 & 70.00 & 76.00 & 0.09 & 0.09 & 0.18 \\
$110 \times 10$ & 0.46 & 2.26 & 9.46 & 0.00 & 75.00 & 100.0 & 0.31 & 0.00 & 0.01 \\
$110 \times 20$ & 2.56 & 3.65 & 11.22 & 20.00 & 90.00 & 100.0 & 0.11 & 0.20 & 0.00 \\
mean & 1.93 & 2.64 & 7.28 & 48.73 & 49.94 & 77.29 & 0.26 & 0.25 & 0.14 \\
\hline
\end{tabular}

Fig. 2 shows the Pareto fronts before and after the local search function is added to the algorithm proposed in this paper. It is shown that the convergence of the algorithm without local search is better than that of the one with local search, indicating that the built-in local search function is enough to meet the calculation requirements and can guide the evolution of the population effectively.

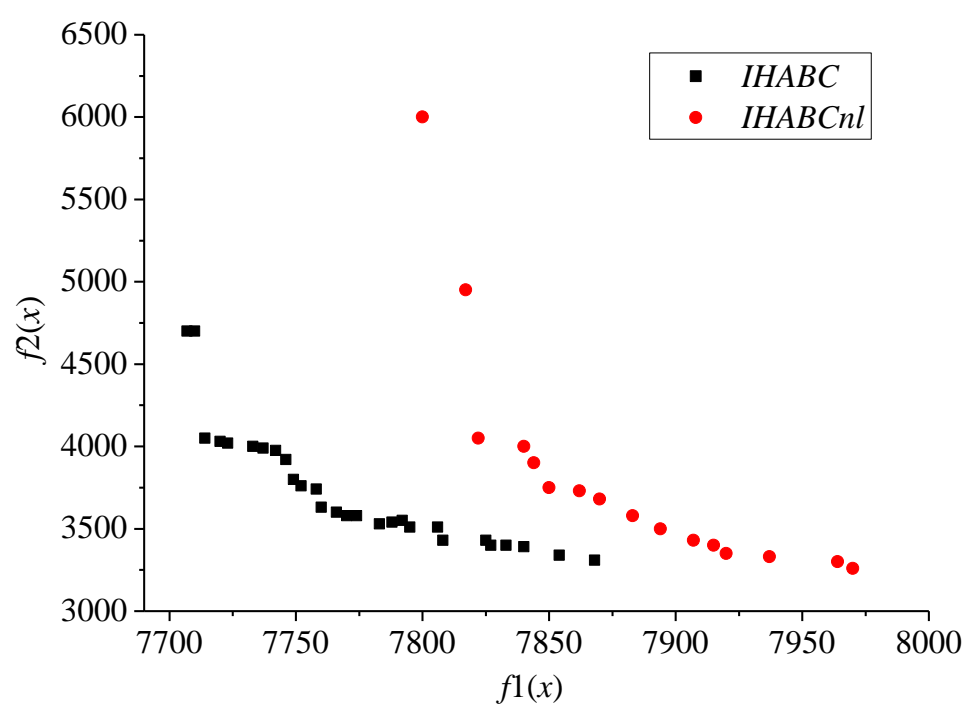

Figure 2: Pareto fronts obtained by IHABC and IHABCnl. 
Fig. 3 shows the $D\left(S_{i}\right)$ evolution curves of TA, INSGA, NGA, BBEDA and the proposed algorithm in the instance of $110 \times 20$ with 1000 iterations. It is shown in the figure that the evolution curve of the proposed algorithm is obviously lower than those of the other four algorithms. It is also shown that the proposed algorithm can effectively guide the evolution of the population along the Pareto direction.

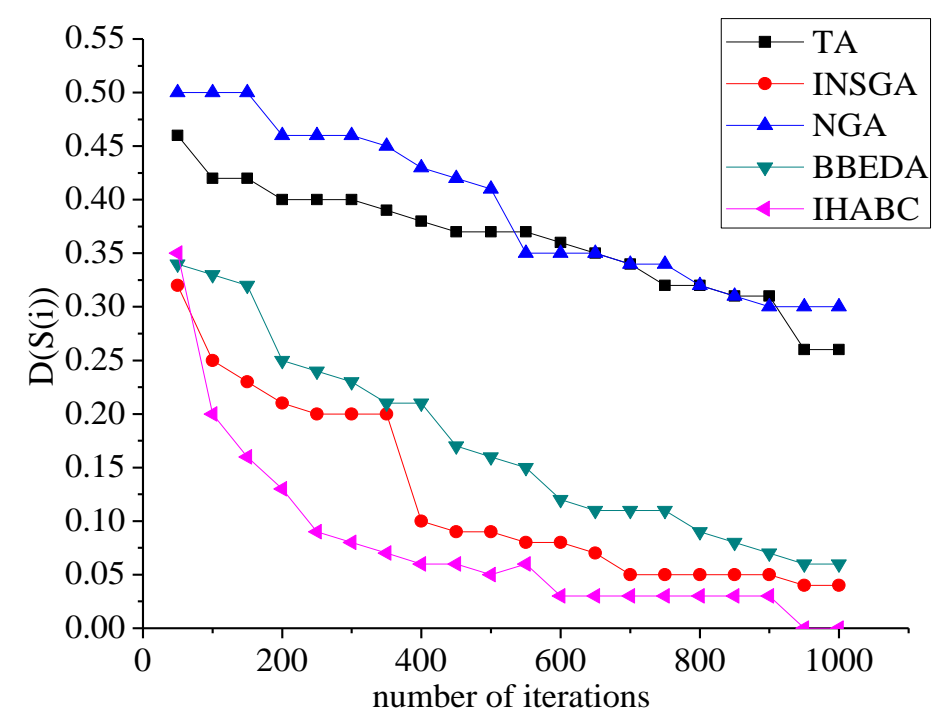

Figure 3: Convergence curves of instances $110 \times 20$.

\section{MULTI-OBJECTIVE FLOW SHOP SCHEDULING CONSIDERING MACHINE FAULTS}

\subsection{Problem description}

The last section discusses the multi-objective batch scheduling when there is any product blockage. In fact, in actual production, machine faults often occur, preventing the entire production line from working and greatly affecting the production efficiency. Therefore, this paper designs the flow shop scheduling against machine faults.

Fig. 4 shows an example of the multi-objective flow shop scheduling considering machine faults. There are two main types of machine faults: one is the faults that are likely to occur to the machines during the wait time or processing time in the beginning of processing, and the second is those faults that only occur when the machines are working. No wear or failure would occur when the machines are not working.

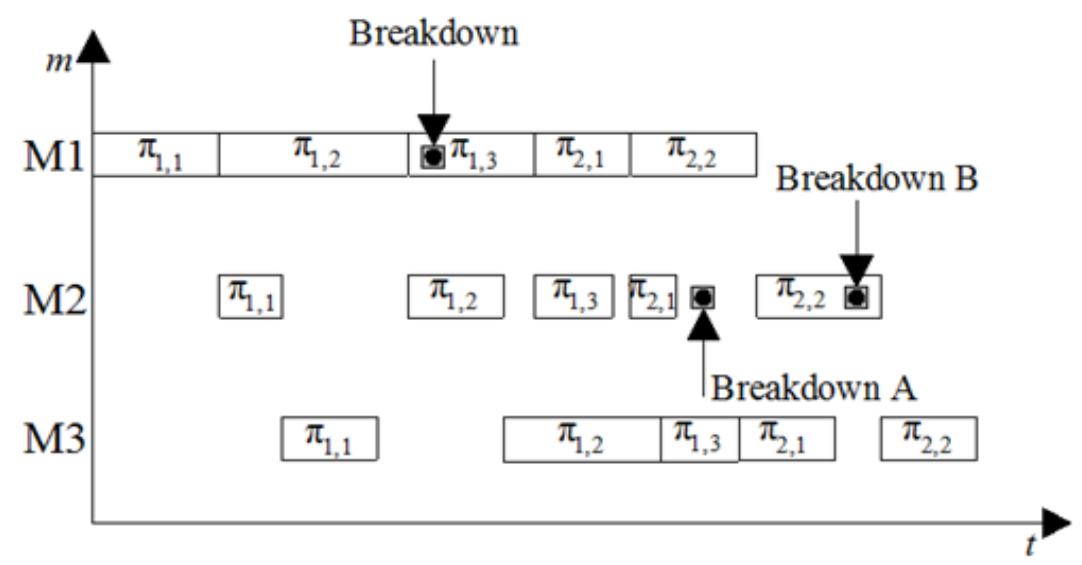

Figure 4: Gantt figure of lot-streaming flow shop with machine breakdown. 
When the machine fault factor is taken into account in the model proposed in this paper, other factors should also be considered. For example, when a machine fails, the product processing on the machine should be stopped immediately. When the machine is repaired, the product should be reworked rather than be processed continually.

The proposed multi-objective flow shop scheduling machine fault is shown in Fig. 5. The main steps are as follows: the steps of the proposed multi-objective flow shop scheduling considering machine faults are shown in Fig. 5 and summarized as follows:

(1) Initialize parameters like population size, $p c$ and calculation time, etc.

(2) Use vNEH to generate a set of populations and assess the maximum completion time and early completion time for each solution within the set;

(3) Add the machine fault factor into the model and recalculate the robustness and stability of each solution in Step (2);

(4) Substitute the calculate $p c$ value into the ISBOX method to work out a new solution and calculate relevant target values;

(5) Determine whether there is any machine having a fault on the assembly line; and if there is, start the re-scheduling;

(6) Carry out the dynamic storage of new solutions. After several iterations, determine whether the termination condition is met.

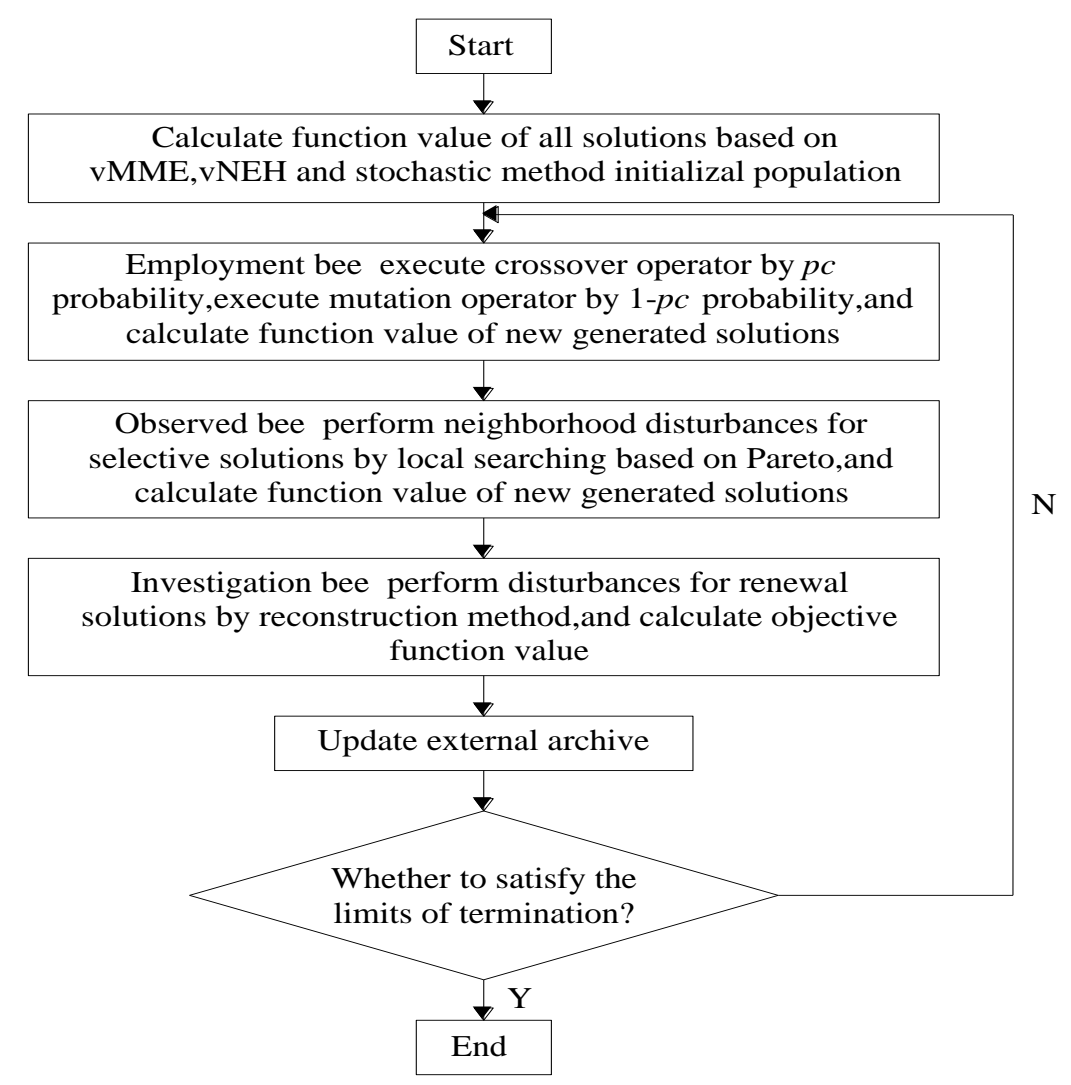

Figure 5: Flowchart of multi-objective blocking lot-streaming flow shop scheduling problem with machine breakdown.

\subsection{Test verification and result analysis}

Using similar test setup as above, this paper verifies the proposed multi-objective batch scheduling model that considers machine faults and compares it with the traditional DABC, INSGA and DHS algorithms.

Fig. 6 shows the evolutionary curves of the $R\left(S_{i}\right)$ index under the NEH and vNEH heuristic methods. It can be seen that both the local and global convergence of vNEH are 
better than those of NEH, which shows that $\mathrm{vNEH}$ is more superior in multi-objective flow shop scheduling, gives new solutions of higher quality and is more helpful to population convergence.

Fig. 7 shows the $D\left(S_{i}\right)$ and $R\left(S_{i}\right)$ values of the proposed algorithm in the case of different crossover operators. From the figure, it can be seen that, when $p c=0, D\left(S_{i}\right)$ is the largest and $R\left(S_{i}\right)$ is the smallest, because when $p c=0$, the model only uses the mutation operator rather than the crossover operator to generate new solutions and at this point, the convergence of the algorithm is the worst; when $p c=0.1-0.9$, the $D$ value gradually decreases and the $R$ value increases gradually. When $p c=1.0$, the $D$ value rises after reaching the minimum value. This is because the algorithm only uses the crossover operator rather than the mutation operator in the evolution process, which leads to local optimal solution. Therefore, finally this paper sets $p c=0.9$.

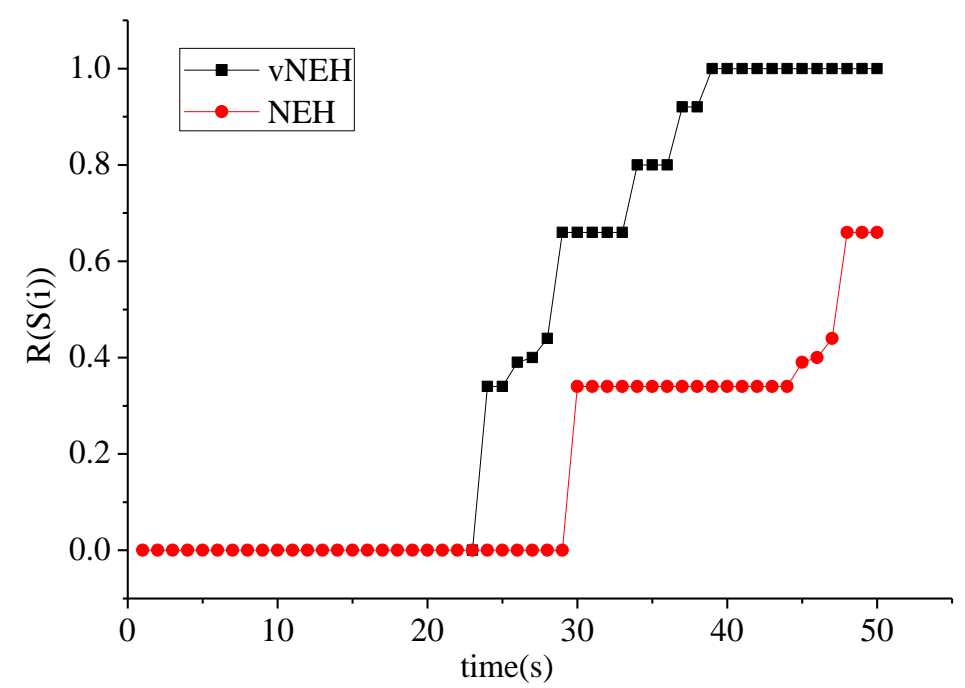

Figure 6: Performance of different initialization strategies.

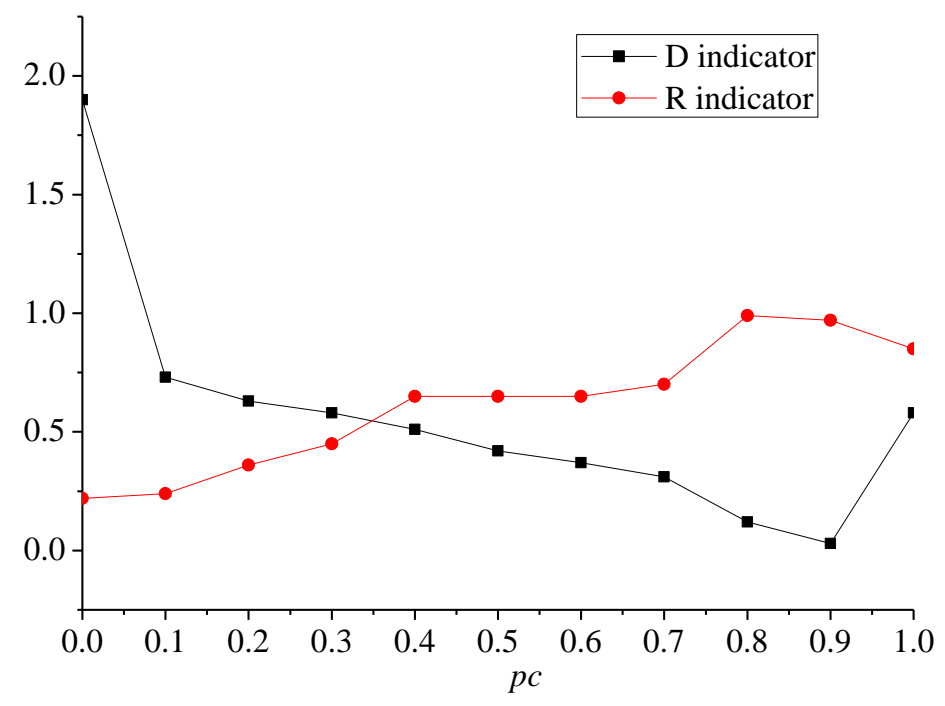

Figure 7: Effects of $p c$ on $D$ and $R$ indicators.

Fig. 8 shows the $D(S i)$ evolution curves of DABC, DHS, INSGA and the proposed algorithm in the instance of $110 \times 15$. Among them, DABC and DHS algorithms do not consider the rescheduling strategy. The number of machine faults on the assembly line is set at 16. It can be seen from the figure that the $D\left(S_{i}\right)$ evolution curve under the proposed algorithm is the lowest, followed by that under the INSGA algorithm. The DABC and DHS 
algorithms that do not consider the re-scheduling strategy have the highest $\left(S_{i}\right)$ evolution curves. This is because the proposed algorithm and the INSGA algorithm utilize the information about nondominated solutions, which increases the probability of producing highquality offspring and direct the population to evolve towards the optimal Pareto. The DABC and DHS algorithms do not take into account the information on nondominated solutions, which reduces the convergence efficiency of the algorithms.

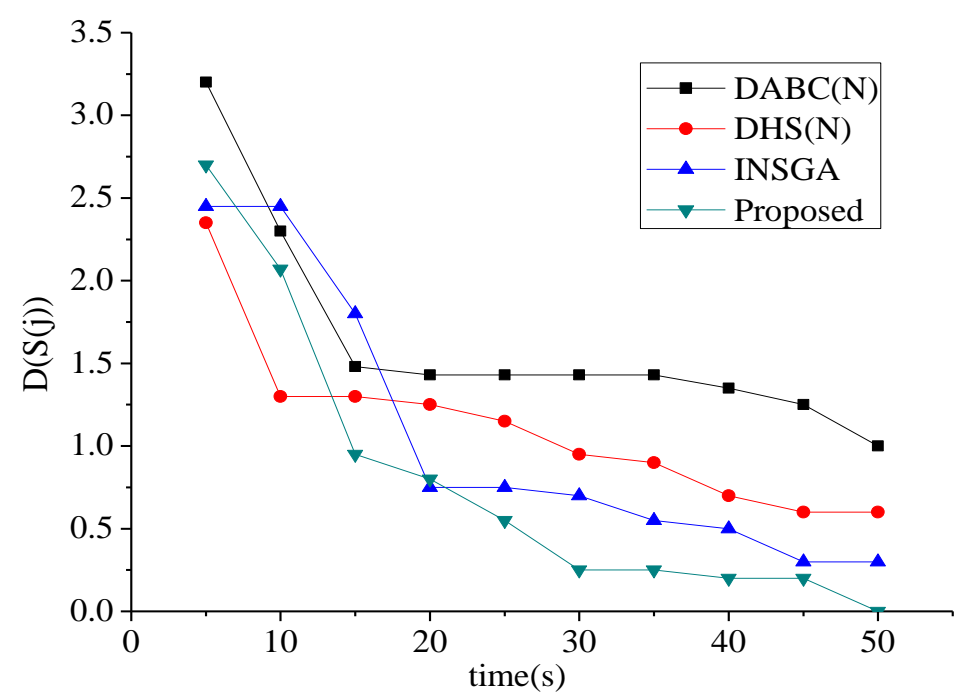

Figure 8: Convergence curves of instances $110 \times 15$.

\section{CONCLUSIONS}

Considering problems like product blockage and machine faults in the batch scheduling of product assembly lines, this paper proposes a multi-objective flow shop batch scheduling model taking these problems into account, and by comparing with the traditional flow shop scheduling model, it verifies the feasibility and superiority of this model. The conclusions are summarized as follows:

(1) Regarding the product blockage problem, this paper improves the MWH heuristic algorithm, leading to better quality of the initial population produced; and that it also proposes a new ISBOX crossover operator algorithm, which is used in combination with the nondominated solution information to calculate the new solution for offsprings, improving the local search capability of the algorithm.

(2) In order to address machine faults, this paper obtains new solutions by optimizing the maximum completion time and the early completion time, takes the new solutions as the initial population, and uses the proposed model to optimize the robustness and stability of the existing algorithm.

(3) By comparing the proposed model and the traditional scheduling model in terms of product blockage and machine fault, this paper finds that the proposed algorithm has high local and global convergence, and generates high-quality nondominated solutions. According to the re-scheduling strategy, this algorithm can effectively reduce the effects of product blockage and machine faults on the objective function.

\section{ACKNOWLEDGEMENT}

Supported by Natural Science Foundation of P. R. China (No. 61503316, 61373147), Fujian Province Science and Technology Plan Project (No. 2016I0015, 2016Y0079), Xiamen Science and Technology Plan of University Innovation Project (No. 3502Z20153020). 


\section{REFERENCES}

[1] Logendran, R.; DeSzoeke, P.; Barnard, F. (2006). Sequence-dependent group scheduling problems in flexible flow shops, International Journal of Production Economics, Vol. 102, No. 1, 66-86, doi:10.1016/j.ijpe.2005.02.006

[2] Wang, S.; Sarker, B. R. (2006). Optimal models for a multi-stage supply chain system controlled by kanban under just-in-time philosophy, European Journal of Operational Research, Vol. 172, No. 1, 179-200, doi:10.1016/j.ejor.2004.10.001

[3] Sarker, B. R.; Balan, C. V. (1999). Operations planning for a multi-stage kanban system, European Journal of Operational Research, Vol. 112, No. 2, 284-303, doi:10.1016/s03772217(97)00432-3

[4] Junior, M. L.; Filho, M. G. (2010). Variations of the kanban system: literature review and classification, International Journal of Production Economics, Vol. 125, No. 1, 13-21, doi:10.1016/j.ijpe.2010.01.009

[5] Al-Tahat, M. D.; Mukattash, A. M. (2006). Design and analysis of production control scheme for kanban-based JIT environment, Journal of the Franklin Institute, Vol. 343, No. 4-5, 521-531, doi:10.1016/j.jfranklin.2006.01.001

[6] Pettersen, J.-A.; Segerstedt, A. (2009). Restricted work-in-process: a study of differences between kanban and CONWIP, International Journal of Production Economics, Vol. 118, No. 1, 199-207, doi:10.1016/j.ijpe.2008.08.043

[7] Hejazi, S. R.; Saghafian, S. (2005). Flowshop-scheduling problems with makespan criterion: a review, International Journal of Production Research, Vol. 43, No. 14, 2895-2929, doi: $10.1080 / 0020754050056417$

[8] Kurihara, K.; Li, Y. L.; Nishiuchi, N.; Masuda, K. (2009). Flow shop scheduling for separation model of set-up and net process based on Branch-and-Bound method, Computers \& Industrial Engineering, Vol. 57, No. 2, 550-562, doi:10.1016/j.cie.2008.08.004

[9] Pan, Q. K.; Wang, L.; Qian, B. (2009). A novel differential evolution algorithm for bi-criteria nowait flow shop scheduling problems, Computers \& Operations Research, Vol. 36, No. 8, 24982511, doi:10.1016/j.cor.2008.10.008

[10] Zhang, H.; Zhu, Y.; Zou, W.; Yan, X. (2012). A hybrid multi-objective artificial bee colony algorithm for burdening optimization of copper strip production, Applied Mathematical Modelling, Vol. 36, No. 6, 2578-2591, doi:10.1016/j.apm.2011.09.041

[11] Akbari, R.; Hedayatzadeh, R.; Ziarati, K.; Hassanizadeh, B. (2012). A multi-objective artificial bee colony algorithm, Swarm \& Evolutionary Computation, Vol. 2, 39-52, doi:10.1016/j.swevo. 2011.08.001

[12] Khorsandi, A.; Hosseinian, S. H.; Ghazanfari, A. (2013). Modified artificial bee colony algorithm based on fuzzy multi-objective technique for optimal power flow problem, Electric Power Systems Research, Vol. 95, 206-213, doi:10.1016/j.epsr.2012.09.002

[13] Li, J. Q.; Pan, Q. K.; Gao, K. Z. (2011). Pareto-based discrete artificial bee colony algorithm for multi-objective flexible job shop scheduling problems, The International Journal of Advanced Manufacturing Technology, Vol. 55, No. 9-12, 1159-1169, doi:10.1007/s00170-010-3140-2

[14] Wang, L.; Zhou, G.; Xu, Y.; Liu, M. (2012). An enhanced Pareto-based artificial bee colony algorithm for the multi-objective flexible job-shop scheduling, The International Journal of Advanced Manufacturing Technology, Vol. 60, No. 9-12, 1111-1123, doi:10.1007/s00170-011$\underline{3665-\mathrm{Z}}$

[15] Ruiz, R.; Maroto, C.; Alcaraz, J. (2006). Two new robust genetic algorithms for the flowshop scheduling problem, Omega, Vol. 34, No. 5, 461-476, doi:10.1016/j.omega.2004.12.006

[16] Xiong, J.; Xing, L. N.; Chen, Y. W. (2013). Robust scheduling for multi-objective flexible jobshop problems with random machine breakdowns, International Journal of Production Economics, Vol. 141, No. 1, 112-126, doi:10.1016/j.ijpe.2012.04.015

[17] Alcaide, D.; Rodriguez-Gonzalez, A.; Sicilia, J. (2002). An approach to solve the minimum expected makespan flow-shop problem subject to breakdowns, European Journal of Operational Research, Vol. 141, No. 2, 384-398, doi:10.1016/s0377-2217(02)00077-2 
[18] Wang, K.; Choi, S. H. (2012). A decomposition-based approach to flexible flow shop scheduling under machine breakdown, International Journal of Production Research, Vol. 50, No. 1, 215234, doi:10.1080/00207543.2011.571456

[19] Alrabghi, A.; Tiwari, A. (2016). A novel framework for simulation-based optimisation of maintenance systems, International Journal of Simulation Modelling, Vol. 15, No. 1, 16-28, doi:10.2507/ijsimm15(1)2.316

[20] Li, M.; Wu, C.; Zhang, L.; You, L.-N. (2015). An intuitionistic fuzzy-TODIM method to solve distributor evaluation and selection problem, International Journal of Simulation Modelling, Vol. 14, No. 3, 511-524, doi:10.2507/ijsimm14(3)co12

[21] Naderi, B.; Zandieh, M.; Roshanaei, V. (2009). Scheduling hybrid flowshops with sequence dependent setup times to minimize makespan and maximum tardiness, The International Journal of Advanced Manufacturing Technology, Vol. 41, No. 11-12, 1186-1198, doi:10.1007/s00170008-1569-3

[22] Zandieh, M.; Ghomi, S. M. T. F.; Husseini, S. M. M. (2006). An immune algorithm approach to hybrid flow shops scheduling with sequence-dependent setup times, Applied Mathematics and Computation, Vol. 180, No. 1, 111-127, doi:10.1016/j.amc.2005.11.136

[23] Lei, D. (2011). Scheduling stochastic job shop subject to random breakdown to minimize makespan, The International Journal of Advanced Manufacturing Technology, Vol. 55, No. 9-12, 1183-1192, doi:10.1007/s00170-010-3151-z

[24] Huang, C. S.; Huang, Y. C.; Lai, P. J. (2012). Modified genetic algorithms for solving fuzzy flow shop scheduling problems and their implementation with CUDA, Expert Systems with Applications, Vol. 39, No. 5, 4999-5005, doi:10.1016/j.eswa.2011.10.013

[25] Sotskov, Y. N.; Lai, T. C.; Werner, F. (2013). Measures of problem uncertainty for scheduling with interval processing times, OR Spectrum, Vol. 35, No. 3, 659-689, doi:10.1007/s00291-012$\underline{0306-3}$

[26] Xue, P.; Zhang, Y.; Yu, X. (2014). Single-machine scheduling with piece-rate maintenance and interval constrained position-dependent processing times, Applied Mathematics and Computation, Vol. 226, 415-422, doi:10.1016/j.amc.2013.10.034 